\title{
Molecular and Biochemical Characterization of Opisthorchis viverrini Calreticulin
}

\author{
Wanlapa Chaibangyang', Amornrat Geadkaew-Krenc', Suksiri Vichasri-Grams², Smarn Tesana ${ }^{3}$, Rudi Grams 1,* \\ ${ }^{1}$ Graduate Program in Biomedical Sciences, Faculty of Allied Health Sciences, Thammasat University, Pathumthani 12121, Thailand; ' $D$ Department of \\ Biology, Faculty of Science, Mahidol University, Bangkok 10400, Thailand; ${ }^{3}$ Food-borne Parasite Research Group, Department of Parasitology, \\ Faculty of Medicine, Khon Kaen University, Khon Kaen 40002, Thailand
}

\begin{abstract}
Calreticulin (CALR), a multifunctional protein thoroughly researched in mammals, comprises N-, P-, and C-domain and has roles in calcium homeostasis, chaperoning, clearance of apoptotic cells, cell adhesion, and also angiogenesis. In this study, the spatial and temporal expression patterns of the Opisthorchis viverrini CALR gene were analyzed, and calcium-binding and chaperoning properties of recombinant O. viverrini CALR (OvCALR) investigated. OvCALR mRNA was detected from the newly excysted juvenile to the mature parasite by RT-PCR while specific antibodies showed a wide distribution of the protein. OvCALR was localized in tegumental cell bodies, testes, ovary, eggs, Mehlis' gland, prostate gland, and vitelline cells of the mature parasite. Recombinant OvCALR showed an in vitro suppressive effect on the thermal aggregation of citrate synthase. The recombinant OvCALR C-domain showed a mobility shift in native gel electrophoresis in the presence of calcium. The results imply that OvCALR has comparable function to the mammalian homolog as a calcium-binding molecular chaperone. Inferred from the observed strong immunostaining of the reproductive tissues, OvCALR should be important for reproduction and might be an interesting target to disrupt parasite fecundity. Transacetylase activity of OvCALR as reported for calreticulin of Haemonchus contortus could not be observed.
\end{abstract}

Key words: Opisthorchis viverrini, Platyhelminthes, calreticulin, calcium-binding, chaperone, transacetylase

\section{INTRODUCTION}

Calreticulin (CALR) was originally identified as an abundant and major calcium-binding protein located in the endoplasmic/sarcoplasmic reticulum in human cells $[1,2]$ where it is retained due to the presence of a C-terminal KDEL retention sequence [3]. It was quickly established as an essential multifunctional protein of the endoplasmic reticulum with important roles in cellular calcium homeostasis and protein folding. However, it has also been found in the cytoplasm, the nucleus, on the cell surface, and released from the cell with effects for example on transcription regulation, cell adhesion and migration (reviewed in [4]). In recent years research has started to unravel the importance of calreticulin in the immune system and cancer [5-7] and it is this multitude of functions that suggests CALR as a significant research target in molecular parasi-

\footnotetext{
- Received 13 November 2017, revised 16 December 2017, accepted 18 December 2017. *Corresponding author (rgrams@tu.ac.th) (C) 2017, Korean Society for Parasitology and Tropical Medicine This is an Open Access article distributed under the terms of the Creative Commons Attribution Non-Commercial License (http://creativecommons.org/licenses/by-nc/4.0) which permits unrestricted non-commercial use, distribution, and reproduction in any medium, provided the original work is properly cited.
}

tology. Indeed, within a short time after its discovery in mammalian cells calreticulin was also identified in human important helminths, blood fluke, and filarial, and Schistosoma mansoni CALR was the first characterized trematode ortholog $[8,9]$. The authors showed calcium-binding of the recombinant protein and demonstrated that the native protein was more abundant in mature worms and eggs compared to $18 \mathrm{hr}$ schistosomula and cercariae. Immunolocalization detected the protein in parenchyma and intestinal epithelia, mature vitelline cells and ova were clearly labeled in females, and in males the testes were positive. The highly conserved ortholog of Schistosoma japonicum was described some years later and its calcium-binding property demonstrated [10,11]. A transacetylase activity of Haemonchus contortus CALR was described $[12,13]$. The present study is a basic characterization of the molecular and biochemical properties of O. viverrini CALR and demonstrates its calcium-binding and chaperoning properties. Spatial distribution suggests that it has important roles throughout the reproductive system of the parasite and that further research should investigate possibilities to decrease parasite fecundity based on inhibition of OvCALR. 


\section{MATERIALS AND METHODS}

\section{Parasites}

O. viverrini metacercariae were isolated from naturally infected cyprinoid fish in the northeast of Thailand. Newly excysted juveniles (NEJ) were obtained by mechanically excysting metacercariae using a disposable needle. Syrian golden hamsters were infected with 50 metacercariae by intragastric intubation. Juvenile and mature parasites were harvested from the biliary system of the infected hamsters at 2, 4, and 8-week postinfection. The collected parasites were washed in $0.85 \%$ $\mathrm{NaCl}$ and kept in liquid nitrogen. All animal experiments in this research were approved by the Animal Ethics Committee of Thammasat University (no. 014/2557) in accordance with the Ethics of Animal Experimentation of the National Research Council of Thailand.

\section{Molecular cloning and sequence analysis}

Total RNA was extracted from adult O. viverrini using TRIzol reagent (Invitrogen, Carlsbad, California, USA), treated with DNase (Promega, Madison, Wisconsin, USA) and 100 ng was used for first strand cDNA generation using RevertAid reverse transcriptase (Thermo Scientific, Vilnius, Lithuania) and an oligo (dT) 18 primer. The reverse transcription product was used as template to amplify a cDNA fragment containing the coding sequence of $O$. viverrini calreticulin (OvCALR) by conventional PCR at $55^{\circ} \mathrm{C}$ annealing, $68^{\circ} \mathrm{C}$ extension temperature using the specific forward primer 5'-gct agc GAG GTT TAC TTT TAT GAA C-3' and reverse primer 5'-gcg gcc gcA AGT TCT TCA TGG GTC TTC G-3' based on an uncharacterized OvCALR expressed sequence tag (Ov_Contig3944, [14]).

A cDNA encoding calreticulin of mouse (MmCALR) was cloned by RT-PCR as described above from mouse total RNA using primers designed on database entry GenBank: NM_007591, forward primer 5'-cat atg GAC CCT GCC ATC TAT TTC-3', reverse primer 5'-ctc gag CAG CTC ATC CIT GGC TTG G-3'. The PCR products were inserted into the $\mathrm{PGEM}^{\circledR}$-T Easy vector (Promega) and the nucleic acid sequences of the OvCALR and MmCALR cDNAs were verified by DNA sequencing. The introduced Nhe I and Not I (OvCALR) and Nde I and Xho I (MmCALR) restriction endonuclease recognition sites (lower case) in forward and reverse primers, respectively, were later used to subclone the cDNA fragments into the pET21b $(+)$ vector (Novagen, Darmstadt, Germany) for expression of recombinant protein. Analyses, retrieval, and editing of sequences were done using EMBOSS
6.6 [15], Clustal Omega 1.2.3 [16], SignalP 4.1 [17], PROSITE release 20.128 [18], BLAST services at http://blast.ncbi.nlm.nih. gov/Blast.cgi, UniProt database [19] at http://www.uniprot.org, NCBI nucleotide database at http://www.ncbi.nlm.nih.gov/nucleotide.

\section{Preparation of $O$. viverrini total RNA and RT-PCR}

Total RNA was extracted from NEJ, 2-, 4-, and 8-week-old $O$. viverrini and used for RT-PCR as described above. The specific primers for OvCALR were 5'-CCG GAC AAC AAA TTC AAG GT3', 5'-ATC AAA CAC GGA TCC AGA GG-3'. O. viverrini actin (OvActin) was used as internal control and amplified with primers 5'-TCG AGA GAA GAT GAC ACA GA-3', 5'-GAT ATC ACG CAC GAT TTC TC-3'. The RT-PCR products (OvCALR: 453 bp, OvActin: 292 bp) were resolved on a 1\% agarose gel.

Expression of recombinant OvCALR, mouse CALR in Escherichia coli, and production of rOvCALR antisera in mice

The plasmids pET21b(+)-OvCALR and pET21b(+)-MmCALR were introduced into E. coli Rosetta-gami (DE3) pLysS by standard chemical transformation. Single positive colonies verified by small-scale screening were inoculated in $5 \mathrm{ml}$ Luria-Bertani (LB) medium containing $100 \mu \mathrm{g} / \mathrm{ml}$ ampicillin, $34 \mu \mathrm{g} / \mathrm{ml}$ chloramphenicol, $12.5 \mu \mathrm{g} / \mathrm{ml}$ kanamycin and $3.125 \mu \mathrm{g} / \mathrm{ml}$ tetracycline, and incubated at $37^{\circ} \mathrm{C}$ with shaking, overnight. On the next day, $100 \mathrm{ml} \mathrm{LB}$ medium containing antibiotics as above was inoculated with $2 \mathrm{ml}$ of the overnight culture and grown at $37^{\circ} \mathrm{C}, 250 \mathrm{rpm}$ shaking until an $\mathrm{OD}_{600}$ of 0.6 . The expression was induced by isopropyl- $\beta$-D-1-thiogalactopyranoside (IPTG) (Fermentas, Vilnius, Lithuania) at a final concentration of 1 $\mathrm{mM}$ and the culture continued overnight. The bacterial cells were harvested by centrifugation at $4,000 \mathrm{~g}, 4^{\circ} \mathrm{C}$ for $20 \mathrm{~min}$. The recombinant proteins were purified by Ni-NTA affinity chromatography (QIAGEN, Hilden, Germany) under native conditions following the manufacturer's instruction. The purified proteins were dialyzed against phosphate buffered saline (PBS), pH 7.2 and protein concentration was determined by a Bradford assay (Bio-Rad, Richmond, California, USA).

Three female ICR mice, 6-8 week-old, were intraperitoneally immunized with $10 \mu \mathrm{g}$ rOvCALR per mouse 3 times in a 3 -week interval. The antigen was prepared by mixing equal volumes of rOvCALR and Titer Max Gold Adjuvant (Sigma, St. Louis, Missouri, USA). The pre-immune sera were collected 1 week before immunization as control sera and the final anti- 
sera were collected 2 weeks after the last immunization.

\section{Immunohistochemistry}

Freshly collected adult parasites were fixed by methacarn fixation and embedded in paraffin. The embedded tissue was cut in $8 \mu \mathrm{m}$-sections on a microtome and mounted on gelatincoated microscopic slides. The parasite sections were deparaffinized twice in xylene and rehydrated in a series of decreasing alcohol concentrations. Antigen retrieval was performed by heating the sections in $10 \mathrm{mM}$ sodium citrate, $\mathrm{pH}$ 6.0, 0.05\% Tween 20. Non-specific binding sites were blocked by incubation in $1 \%$ glycine and 4\% BSA in PBS, pH 7.2 each for 30 min at room temperature. After blocking, the parasite sections were incubated with either mouse anti-rOvCALR antiserum or pre-immune serum $(1: 2,000)$ in $1 \% \mathrm{BSA}$ in PBS, $\mathrm{pH} 7.2$ at $4^{\circ} \mathrm{C}$ overnight. Endogenous peroxidase activity was blocked by incubating sections in $3 \% \mathrm{H}_{2} \mathrm{O}_{2}$ (Merck, Hohenbrunn, Germany) in the dark at room temperature twice for $10 \mathrm{~min}$. The sections were then incubated in biotinylated polyclonal rabbit anti-mouse immunoglobulin (1:200) (Dako, Copenhagen, Denmark) at $37^{\circ} \mathrm{C}$ for $1 \mathrm{hr}$. Following washes, the avidin-biotin complex $(\mathrm{ABC})$ peroxidase staining kit (Thermo Scientific, Rockford, Illinois, USA) and the chromogenic substrate 3-amino-9-ethyl carbazole (AEC) (Vector, Burlingame, California, USA) were used for standard colorimetric detection. The reaction was stopped by washing in PBST (10 mM PBS, pH 7.2, $0.1 \%$ Tween 20 ).

\section{Parasite crude worm extract preparation}

Adult parasites were homogenized in 10 mM PBS, pH 7.2, $150 \mathrm{mM} \mathrm{NaCl}, 0.5 \%$ (v/v) Triton X-100, 1 mM PMSF, $1 \mathrm{mM}$ EDTA at $4^{\circ} \mathrm{C}$. The homogenate was centrifuged at $12,000 \mathrm{~g}$, $4^{\circ} \mathrm{C}$ for $15 \mathrm{~min}$ to remove insoluble material. The supernatant was collected as soluble crude worm extract. The pellet was solubilized in $50 \mathrm{mM}$ Tris-HCl, pH 8.0 and 3\% (w/v) SDS at $37^{\circ} \mathrm{C}$ for $1 \mathrm{hr}$ and then centrifuged at $12,000 \mathrm{~g}$ for $15 \mathrm{~min}$. The supernatant was collected as insoluble crude worm extract. The protein concentration of the extracts was measured by a BCA protein assay kit (Thermo Scientific).

\section{SDS-PAGE and western analysis}

Fifteen $\mu \mathrm{g}$ each of soluble and insoluble parasite crude worm extract and 100 ng each of recombinant OvCALR, MmCALR, and SjGST-OvCALR C-domain were size-separated by $12.5 \%$ Tris-glycine SDS-PAGE and transferred to nitrocellu- lose membranes (Bio-Rad) by semi-dry blotting. Unspecific binding sites on the membranes were blocked in 5\% skim milk (Thermo Fisher Scientific) in Tris-buffered saline (TBS, $150 \mathrm{mM} \mathrm{NaCl}, 20 \mathrm{mM}$ Tris-HCl, $\mathrm{pH}$ 7.5) at room temperature for $1 \mathrm{hr}$ with gentle shaking. Mouse anti-rOvCALR antiserum and mouse pre-immune serum were diluted 1:3,000 in 1\% skim milk in TBS. Each membrane was submerged in one of the antibody dilutions and incubated at $4^{\circ} \mathrm{C}$ with gentle shaking, overnight. On the next day, the membranes were washed 3 times for $5 \mathrm{~min}$ each in washing buffer (TBS, 0.05\% Tween-20) and then incubated with goat anti-mouse IgG (whole molecule)-alkaline phosphatase (Sigma) at dilution 1: 30,000 in $1 \%$ skim milk in TBS at room temperature with gentle shaking, $1 \mathrm{hr}$. Following 3 washes in washing buffer for 5 min each, the membranes were equilibrated in detection buffer (0.1 M Tris- $\mathrm{HCl}$, pH 9.5, $0.1 \mathrm{M} \mathrm{NaCl}$, and $50 \mathrm{mM}$ $\mathrm{MgCl}_{2}$ ) for $5 \mathrm{~min}$. Finally, BCIP/NBT substrate (Amresco LLC, Solon, Ohio, USA) was added, and the membranes were incubated in the dark until the signals were obtained.

\section{Citrate synthase aggregation assay}

Citrate synthase aggregation assays have been previously used to demonstrate the chaperoning properties of calreticulin (for example [20]). Citrate synthase (Sigma) at $1 \mu \mathrm{M}$ final concentration was mixed with rOvCALR at final concentrations of $0.25-1 \mu \mathrm{M}$ in PBS, pH 7.2 in a total reaction volume of $200 \mu \mathrm{l}$. Recombinant MmCALR and BSA (Sigma) were used as positive and negative controls, respectively, at $1 \mu \mathrm{M}$ final concentration. The assay was performed in duplicate in a 96-well UVtransparent plate (Thermo Scientific). The samples were incubated at $45^{\circ} \mathrm{C}$ and citrate synthase aggregation was monitored by measuring light scattering at $360 \mathrm{~nm}$, every $10 \mathrm{~min}$ for $1 \mathrm{hr}$ on a Varioskan Flash spectral scanning multimode reader (Thermo Scientific, Waltham, Massachusetts, USA).

\section{Calcium-binding assay of OvCALR C-domain}

The $12 \mathrm{kDa}$ OvCALR C-domain, comprising the C-terminal 106 amino acids, was expressed in E. coli in fusion with S. japonicum $26 \mathrm{kDa}$ GST (SjGST) to analyze its calcium binding activity. The encoding 321 bp cDNA fragment (including the stop codon) was amplified by PCR using the complete OvCALR cDNA as template with forward primer 5 '-gaa ttc GAT GAT ATT GCT CAT GTT G-3' and reverse primer $5^{\prime}$-gcg gcc gcT TAA AGT TCT TCA TGG GTC-3'. The introduced EcoR I and Not I restriction endonuclease recognition sites (lower case) 
were used to subclone the cDNA fragment into pGEX-5X-1 (GE Healthcare). Following verification of the inserted cDNA sequence recombinant SjGST-OvCALR C-domain and SjGST were expressed in transformant $E$. coli BL21 and purified by affinity chromatography on Glutathione-Superflow Resin (Clontech, Palo Alto, California, USA). The concentration of the purified proteins was measured by a Bradford assay (Bio-Rad). The potential calcium-binding of OvCALR was analyzed by a mobility shift assay in native PAGE. Five micrograms of rSjGST-OvCALR C-domain, recombinant FgCaBP1 (positive control, [21]) and rSjGST (negative control) were incubated with either $\mathrm{CaCl}_{2}$ or EDTA at $1 \mathrm{mM}$ final concentration on ice for $20 \mathrm{~min}$. The samples were mixed with sample buffer (60 $\mathrm{mM}$ Tris-HCl, pH 6.8, 10\% glycerol, 0.025\% Bromophenol Blue, $0.5 \%$ DTT) and resolved on $8.5 \%$ polyacrylamide gels. Sample buffer, gel and electrophoresis running buffer $(25 \mathrm{mM}$ Tris, 192 mM glycine, $\mathrm{pH}$ 8.3) were supplemented with either $\mathrm{CaCl}_{2}$ or EDTA at $1 \mathrm{mM}$ final concentration.

\section{OvCALR transacetylase activity assays}

Transacetylase activity of OvCALR was tested by inhibition of GST in the presence of 7-acetoxy-4-methylcoumarin (7AMC, Sigma, Switzerland) as previously described $[22,23]$. Recombinant SjGST (see the above section) at an amount of 0.5 $\mu \mathrm{g}$ was mixed with $2 \mu \mathrm{g}$ rOvCALR or rMmCALR (see the previous section) and $250 \mu \mathrm{M}$ 7-AMC in $0.25 \mathrm{M}$ potassium phosphate buffer, $\mathrm{pH}$ 6.5, 1 mM EDTA at a final reaction volume of $20 \mu \mathrm{l}$. The samples were incubated at $37^{\circ} \mathrm{C}$ and GST activity was measured in triplicate after 15, 30 and 45 min as follows. The samples were mixed with $180 \mu \mathrm{l}$ substrate solution $(1 \mathrm{mM}$ 1-chloro-2,4-dinitrobenzene (CDNB, Sigma-Aldrich, Poole, Dorset, UK), $2 \mathrm{mM}$ reduced glutathione (Serva, Heidelberg, Germany) in $0.25 \mathrm{M}$ potassium phosphate buffer, $\mathrm{pH}$ 6.5). Absorbance at $340 \mathrm{~nm}$ was read every $60 \mathrm{sec}$ for $10 \mathrm{~min}$ on a Varioskan Flash Spectral Scanning Multimode Reader (Thermo Scientific). Parallel measured samples containing either rSjGST alone or rSjGST/7-AMC, rSjGST/rOvCALR, rSjGST/rMmCALR were used to determine GST activity unaffected by calreticulincaused transacetylation. Control samples containing only substrate solution (see above) were used to determine background values at each time point. Linear regression analysis of the background-corrected values was performed in Prism 6 (GraphPad Software, Inc., San Diego, California, USA). Slope values from all measurements were used to calculate relative GST activity.
Western analysis was performed following incubation of $0.5 \mu \mathrm{g} r$ rjGST with or without $2 \mu \mathrm{g}$ OvCALR in the presence of 50-250 $\mu \mathrm{M}$ 7-AMC in $0.25 \mathrm{M}$ potassium phosphate buffer, $\mathrm{pH}$ $6.5,1 \mathrm{mM}$ EDTA at a final reaction volume of $20 \mu \mathrm{l}, 37^{\circ} \mathrm{C}$ for $40 \mathrm{~min}$. The samples were resolved by SDS-PAGE and transferred onto a nitrocellulose membrane as described above. A rabbit anti-acetylated lysine antibody (Cell signaling, Danvers, MA, USA) at dilution 1:1,000 in TBS, pH 7.5, 5\% BSA, 0.1\% Teen-20 was used for detection at $4^{\circ} \mathrm{C}$, overnight. Alkaline phosphatase-conjugated goat anti-rabbit IgG antibody (Dako) at dilution 1:1,000 was used as secondary antibody at room temperature for $1 \mathrm{hr}$. Colorimetric detection was performed with BCIP/NBT substrates as described above.

\section{RESULTS}

\section{Molecular cloning and sequence analysis}

An uncharacterized CDNA encoding O. viverrini calreticulin (OvCALR) was identified by a BLAST search of the parasite's transcriptome data [14] with the described homologs from $S$. mansoni [8] and S. japonicum [11]. Reverse transcription of adult stage RNA was used to amplify the 1,248 bp open reading frame of this cDNA. The confirmed sequence encoded the complete O. viverrini pre-calreticulin (415 amino acids) carrying a 16 residues signal peptide (SignalP 4.1). The mature protein has a calculated molecular weight of $46.2 \mathrm{kDa}$ and an isoelectric point of 4.3. Sequence conservation is high to the ortholog in Clonorchis sinensis at $96.6 \%$ identity but significantly less to S. mansoni (57.7\%), S. japonicum (53.6\%), and mammalian calreticulin (human 50.9\%, mouse 50.7\%). Decreased sequence conservation between these proteins can be observed towards their $\mathrm{N}$ - and C-termini (Fig. 1). The 3 characteristic domains which comprise the major structural and functional features of calreticulin are present. Residues in the globular Nterminal domain that have been shown to play a role in the chaperoning property of calreticulin are conserved as are the disulfide bond forming cysteines in this domain. The 2 tandem repeated motifs of the arm-forming proline-rich P-domain are present in 3 copies each. The binding region of protein disulfide-isomerase ERp57 at the tip of the P-domain is highly conserved, and the acidic $\mathrm{C}$-domain carries a consensus sequence-fitting (PROSITE: ER_TARGET) terminal endoplasmic reticulum retention signal (HEEL, Fig. 1).

Recent publication of the parasite genome data [24] allowed to compare the exon/intron organization of the human and 


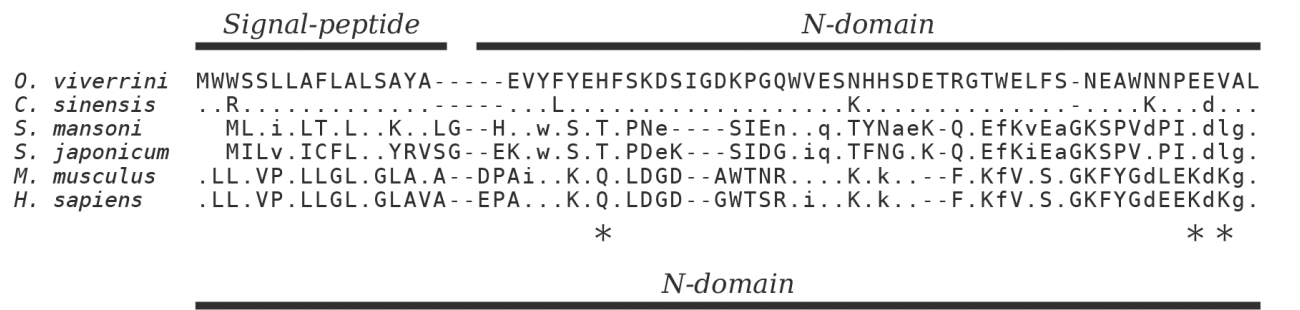

66
66
63
64
66
66
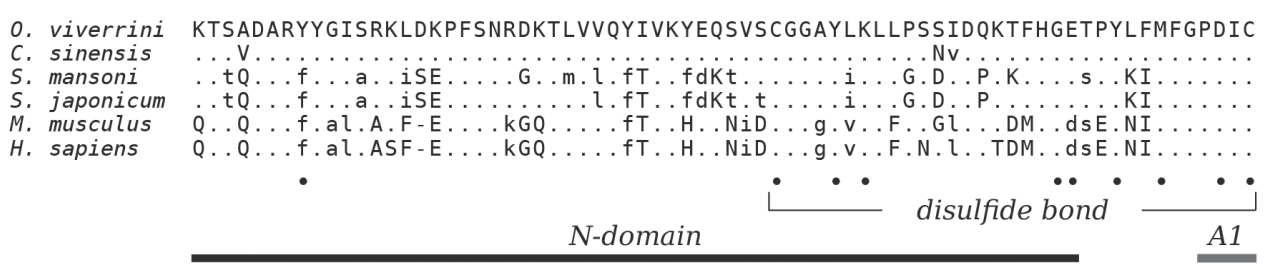

\section{8}

138 135 136 137

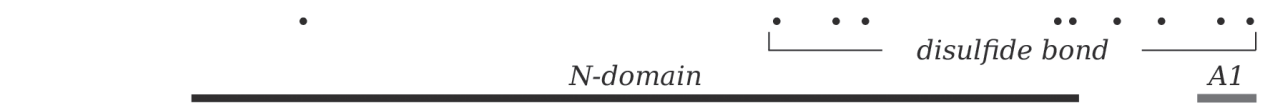

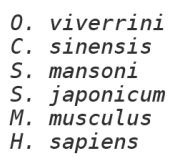

GYSTKKVHAIFSYKGKNHLIKKEVRCKDDLVSHLYTFILTPDNKFKVLIDNEQVEEGSLEDDWDMLLPKEID

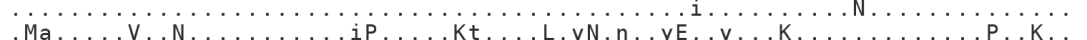

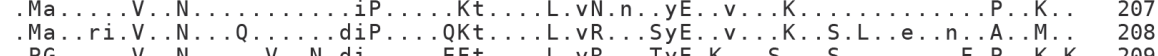

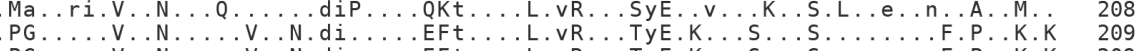
H. sapiens

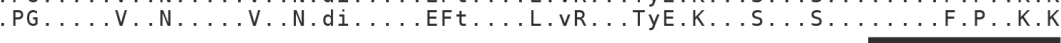
-

$$
\text { - }
$$

$$
\text { A1 }
$$$$
\text { A2 }
$$

A3

P-domain

o. viverrini

C. sinensis

S. mansoni

S. japonicum

M. musculus

H. sapiens

DPASKKPEDWVDEEEIDDPDDKKPEDWDKPKDIPDPDAKKPEDWDDEMDGEWERPKIPNPEYKGDWHPRKIA

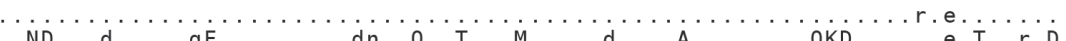

ND . . . .

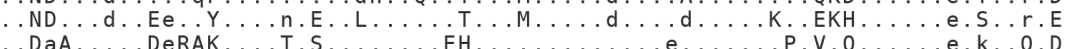

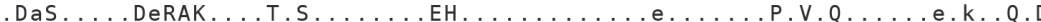

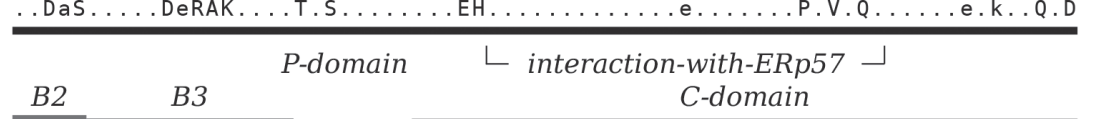

P-domain

\section{C-domain}
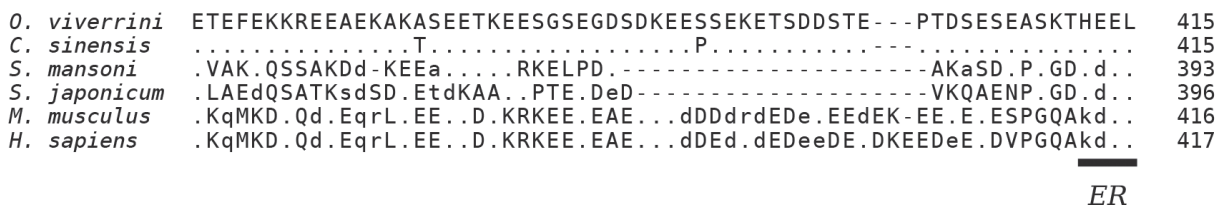

Fig. 1. Multiple alignment of the amino acid sequences of calreticulin from Homo sapiens UniProt: P27797, Mus musculus UniProt: P14211, Opisthorchis viverrini (this publication, UniProt: A0A1P8P1U1), Clonorchis sinensis UniProt: K7NB00, Schistosoma mansoni UniProt: Q06814, Schistosoma japonicum UniProt: O45034. The 3 major regions, N-, P-, and C-domain are indicated based on experimental data of human calreticulin as are N-terminal signal peptide and $\mathrm{C}$-terminal ER signal (KDEL). The 3 each of $\mathrm{A}$ and $\mathrm{B}$ repeats of the P-domain are indicated and the disulfide bond-forming cysteine residues in the $\mathrm{N}$-domain. Residues forming the high-affinity calcium-binding site in human calreticulin [29,30] are indicated by star symbols, and residues important for carbohydrate-binding are indicated by closed circles [29]. The region involved in interaction with oxidoreductase ERp57 is indicated [39]. Amino acids identical, similar, and non-conserved to OvCALR are indicated by dots, lower, and upper case characters, respectively. Gaps, introduced for best alignment are indicated by dashes (-).

parasite CALR genes. The coding sequence of OvCALR is contained in a 1,371 bp fragment of genomic DNA (nucleotides 39198-37828 of GenBank no. JACJ01026242.1) that carries 3 small introns of 46,39 , and 38 bp size at positions $80-125$,
447-485, and 716-753, respectively. Intron 1 separates the signal peptide encoding sequence from the remaining sequence, and an intron at this position is also present in the human CALR gene. Likewise, the position of intron 3 is conserved be- 
tween the parasite and human genes. Intron 2 is only found in the parasite gene while the human gene is interrupted by 6 additional introns not present in the parasite gene.

\section{Expression of OvCALR RNA}

OvCALR RNA was present in NEJ, 2-, 4-, and 8-week-old parasites (Fig. 2). The control, OvActin showed constant expression across the analyzed stages while OvCALR was clearly less abundant in NEJ.

\section{Western analysis of OvCALR}

Recombinant OvCALR, MmCALR, and SjGST-OvCALR Cdomain were expressed in soluble form in Escherichia coli and purified by affinity chromatography (Fig. 3A). Recombinant OvCALR was used to produce antigen-specific polyclonal antisera in mice. Western analysis showed that anti-rOvCALR antiserum was not cross-reactive to rMmCALR while it detected the rOvCALR and rSjGST-OvCALR C-domain (Fig. 3B). Unlike the related calnexin which is a membrane-bound chaperone, calreticulin is a soluble chaperone of the ER. Accordingly, na-

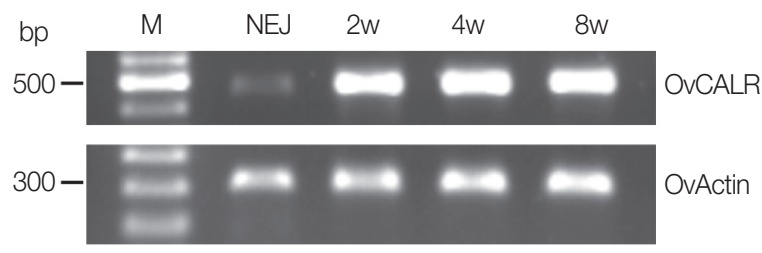

Fig. 2. Reverse transcription PCR analysis of OvCALR with total RNA from newly excysted juveniles (NEJ), 2-, 4-, and 8-week-old parasites $(2 \mathrm{w}, 4 \mathrm{w}$, and $8 \mathrm{w})$. O. viverrini actin RNA was reverse transcribed (OvActin) and used as the control. tive OvCALR was readily detected in soluble crude worm extract and faintly in the insoluble crude worm preparation. A slow gel migration at around $55 \mathrm{kDa}$ has been observed and discussed in early analyses of mammalian calreticulin (for example in [1]). Likewise, OvCALR and native OvCALR migrated slower than expected from their calculated molecular weight comparable to rMmCALR. The mouse pre-immune sera did not show any reactivity to recombinant and native proteins (Fig. 3C).

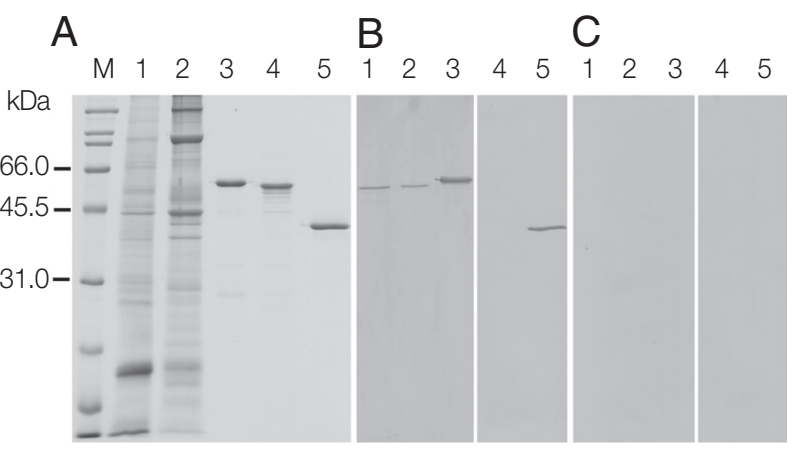

Fig. 3. SDS-PAGE and western analysis of parasite extracts (CW) and recombinant calreticulin. (A) Coomassie Blue R stained SDSPAGE; (B, C) western blots detected with mouse anti-rOvCALR antiserum and mouse pre-immune serum at dilution 1:3,000, respectively. M, broad range protein standard marker (Bio-Rad); Lane 1, soluble CW (15 $\mu \mathrm{g})$; Lane 2, insoluble CW (15 $\mu \mathrm{g}) ;$ Lane 3, purified rOvCALR; Lane 4, purified rMmCALR; Lane 5, purified rSjGST-OVCALR-C domain. Lanes 3-5, 1 $\mu \mathrm{g}$ recombinant protein for Coomassie Blue R staining, 100 ng for antibody detection.

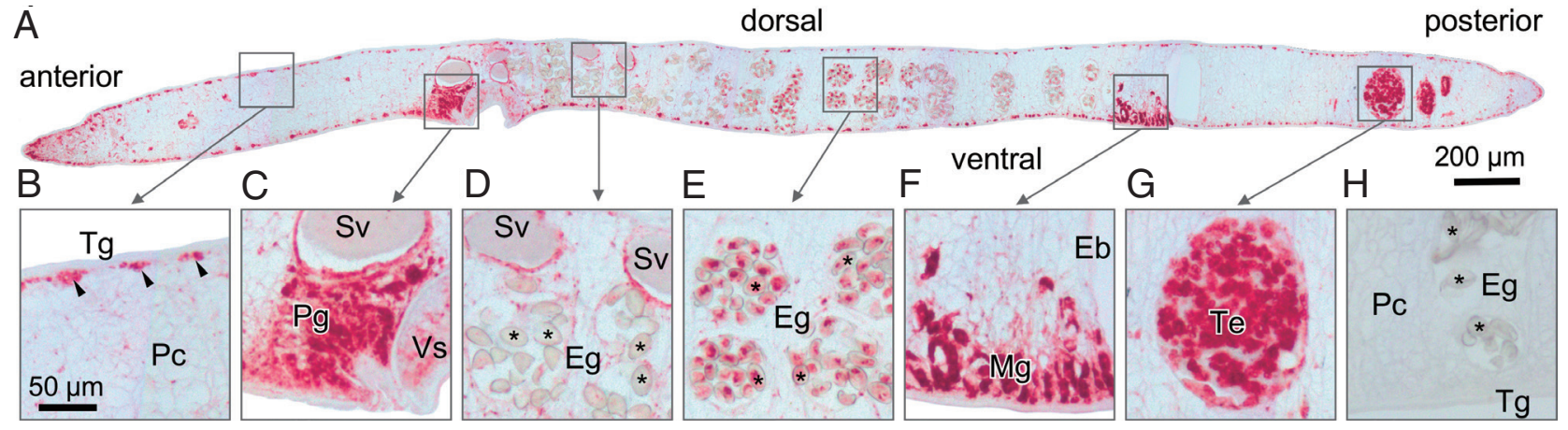

Fig. 4. Immunohistochemical localization of OVCALR in a sagittal section of adult O. viverrini. (A) an overview at low magnification with boxes indicating the magnified regions shown in (B-G); (B) tegumental cell bodies are stained (arrow heads), punctate staining in the parenchyma $(\mathrm{Pc})$, tegument $(\mathrm{Tg})$, and the muscles beneath are unstained; $(\mathrm{C})$ strong staining of prostate gland cells and lining of seminal vesicle; (D) staining is mostly absent in eggs $\left(\mathrm{Eg},{ }^{*}\right)$ in the distal uterus; $(\mathrm{E})$ eggs $\left(\mathrm{Eg},{ }^{*}\right)$ in the proximal half of the uterus show staining; (F) Mehlis' gland (Mg) cells showing strong staining, excretory bladder (Eb) is unstained; (G) strong staining is observed in spermatocytes in the testis; $(H)$ control probed with mouse pre-immune serum. 
Tissue distribution of OvCALR in adult parasites

Sections prepared from paraffin-embedded mature parasites were probed with anti-rOvCALR antiserum. Immunostaining was obvious in the tegumental cell bodies but did not extend to the tegumental syncytium and was also absent in the beneath located muscle layers while the parenchyma showed only punctate staining suggesting the presence of only small amounts of OvCALR in the endoplasmic reticulum. The cecal epithelium was stained. The most intense staining was observed in several parts of the reproductive system, in testes and ovary, prostate gland and Mehlis' gland, and vitelline cells. In the eggs, staining intensity during embryogenesis decreased towards the distal uterus (Fig. 4).

\section{Calcium-binding of rOvCALR C-domain}

In mammals, the C-domain of calreticulin has a high content of acidic residues (42.6\% in HsCALR) and has been characterized as a high capacity/low affinity calcium-binding domain [25]. In comparison the OvCALR C-domain has a reduced number of acidic residues (33.0\%) and its sequence conservation to HsCALR is low at only 33\% identity. To test whether the OvCALR C-domain was still able to bind calcium the recombinant domain in fusion with $S$. japonicum glutathione S-transferase (SjGST) was incubated with either calcium or EDTA and resolved by native PAGE in the presence of either calcium or EDTA in the running buffer (Fig. 5). SjGST was used

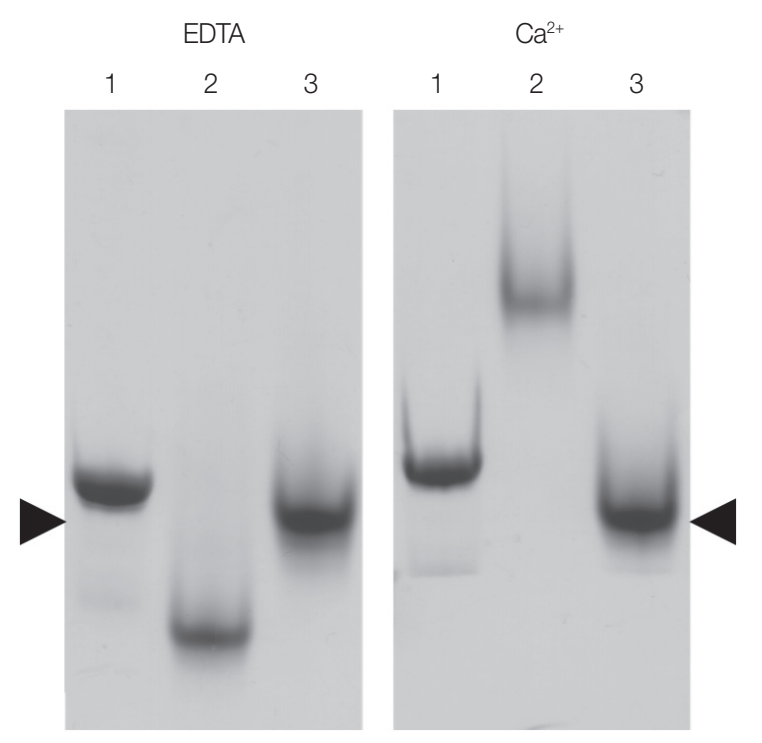

Fig. 5. Native SDS-PAGE showing shift in the migration of OvCALR in the presence of calcium. Lane 1, rOvCALR; Lane 2, positive control rFgCaBP1; Lane 3, negative control rSjGST. Position of rSjGST indicated by arrow heads. as negative control and the EF-hand motif containing Fasciola gigantica calcium-binding protein 1 (FgCaBP1, [21]) was used as positive control. The observed shift in the migration of the OvCALR C-domain demonstrates its ability to bind calcium. Unlike FgCaBP1 with its high affinity calcium-binding EFhands the OvCALR C-domain requires the presence of calcium in the running buffer to observe this effect which supports the low affinity binding observed in mammalian calreticulin.

\section{Chaperoning activity of rOvCALR}

Folding and stabilization of proteins in the ER is a major function of calreticulin. A protein aggregation assay based on citrate synthase has been used to demonstrate the protective function of calreticulin from diverse organisms $[20,26,27]$. The results of this in vitro assay demonstrate that rOvCALR has similar protective function as rMmCALR suppressing aggregation of the enzyme at higher temperature (Fig. 6).

\section{Transacetylase activity of OvCALR}

Transacetylase activity as previously reported for calreticulin of $H$. contortus $[12,13]$ was analyzed using 7-acetoxy-4-methylcoumarin (7-AMC) as substrate [28] for OvCALR-mediated modification of rSjGST. Recombinant OvCALR and rMmCALR suppressed GST activity without 7-AMC while 7-AMC alone reduced GST activity at a lesser amount (Fig. 7A). The combination of calreticulin and 7-AMC appeared to show additive suppressive activity. Further investigation by western analysis with an anti-acetylated lysine antibody demonstrated acetyla-

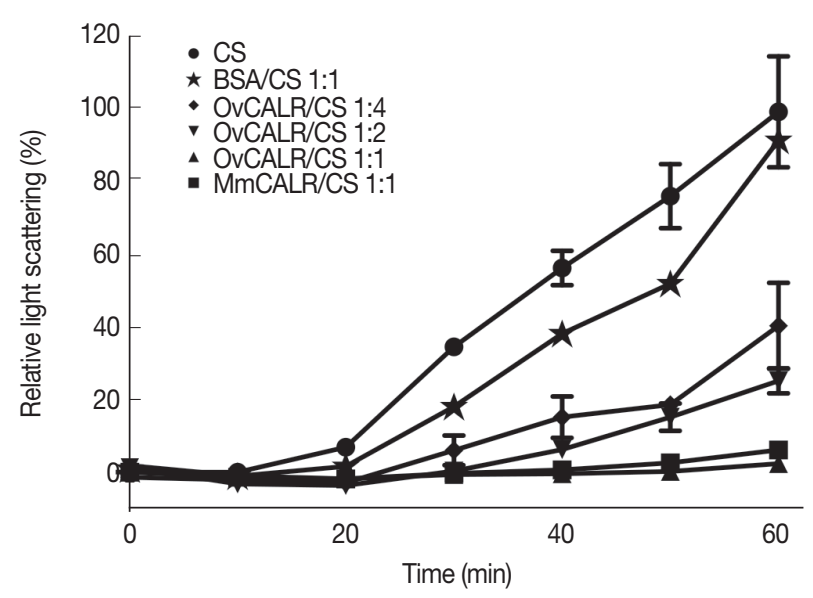

Fig. 6. Line graph showing protection of citrate synthase (CS) against heat-induced aggregation in the presence of rOvCALR. CS was incubated with/without rOvCALR at different molar ratios. Incubation with BSA and recombinant mouse calreticulin (MmCALR) served as negative and positive control, respectively. 

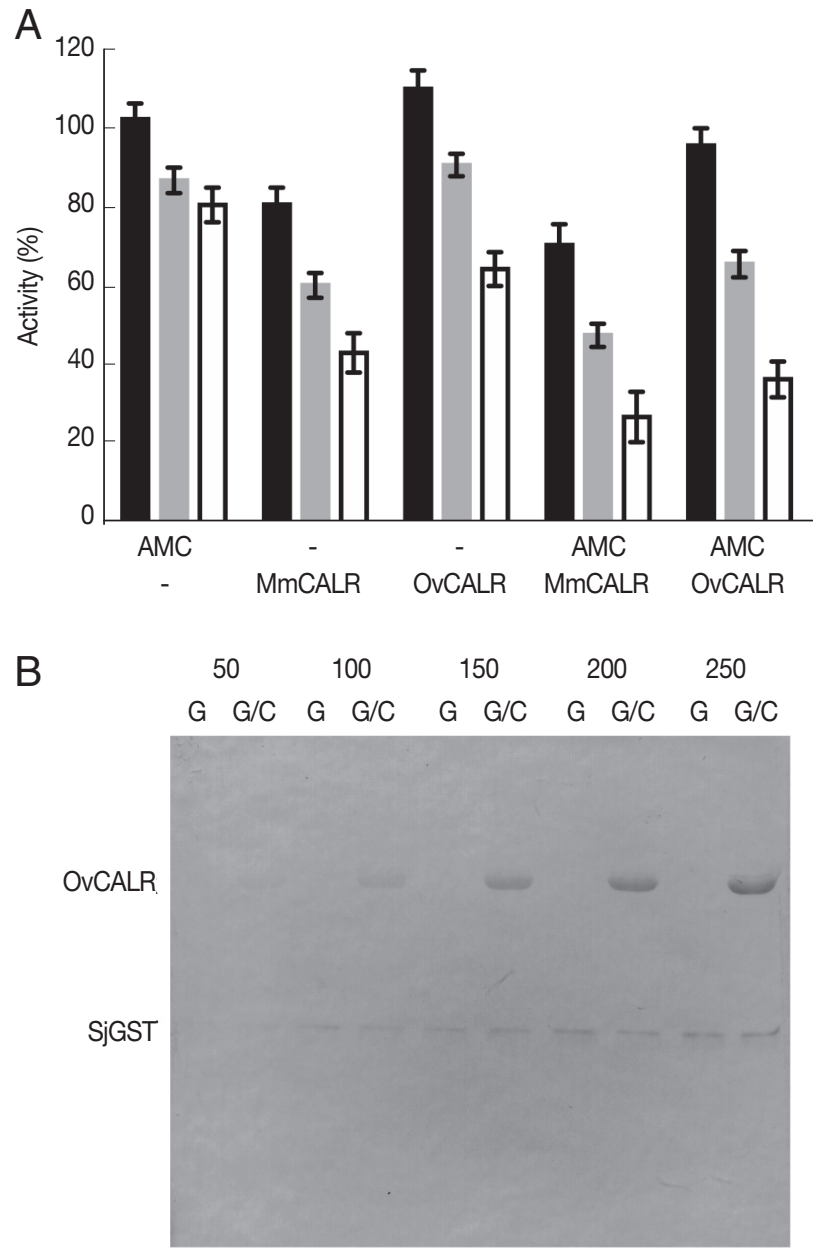

Fig. 7. Determination of OVCALR transacetylase activity. (A) GST activity assay, rSjGST was incubated alone or with the indicated combinations of 7 -AMC $(150 \mu \mathrm{M})$ and recombinant mouse or parasite calreticulin. GST activity was measured at 15, 30, and 45 min (black, gray, and white bars, respectively). Relative remaining activity compared to GST alone at each time point (triplicate measurements with $\mathrm{SD}$ ) is shown. (B) western detection of recombinant OvCALR ( $2 \mu \mathrm{g} / \mathrm{lane})$ and SjGST (0.5 $\mu \mathrm{g} / \mathrm{lane})$ with an anti-acetylated lysine antibody after incubation with 50-250 $\mu \mathrm{M}$ 7-AMC for 40 min. G indicates incubation of SjGST alone, G/C indicates co-incubation of SjGST and OvCALR.

tion of GST by 7-AMC in the absence of calreticulin (Fig. 7B). Direct acetylation through 7-AMC was also observed for BSA and a recombinant $F$. gigantica fatty acid binding protein (data not shown).

\section{DISCUSSION}

The present work demonstrates that OvCALR has basic properties comparable to human calreticulin, i.e., calcium- binding and chaperoning. The high-capacity but low-affinity $\mathrm{Ca}^{2+}$-binding property of the OvCALR C-domain was shown (Fig. 5) but not the high-affinity $\mathrm{Ca}^{2+}$-binding in the site formed by residues Q26, K62, K64, and D328 in human calreticulin (Fig. 1). The latter site was detected in structural analyses of calreticulin $[29,30]$ and confirmed previous data for human calnexin in which residues S75 (Q26 in HsCALR), D118 (D63 in HsCALR), K119 (K64 in HsCALR), and D437 (D328 in HsCALR) were implicated in $\mathrm{Ca}^{2+}$-binding [31]. This site is supposedly important for structural stability of the 2 proteins, and the trematode calreticulins show conservation of the involved acidic residues (Fig. 1) suggesting that they, too, bind a calcium ion in this site.

Calreticulin is known as a soluble endoplasmic lectin that interacts with monoglucosylated oligosaccharides of glycoproteins during folding, but it can also interact and protect nonglycosylated proteins. High sequence conservation of residues involved in carbohydrate-binding (Fig. 1) [29] suggests that OvCALR and other trematode orthologs have the same ability. The citrate synthase assay demonstrated the ability of OvCALR to bind non-glycosylated proteins and to protect them from aggregation (Fig. 6). The binding site for non-glycosylated proteins has been mapped by deletion-analysis to the globular lectin domain using the same assay [27] indicating correct folding of rOVCALR in the required region of the $\mathrm{N}$-domain.

As can be expected from the high conservation of the protein disulfide-isomerase-binding site in the P-domain homologous ERp57-like proteins can be found among the uncharacterized proteins of O. viverrini (UniProt no. A0A095A463) and other trematodes, e.g., S. mansoni (UniProt no. P38658) suggesting conservation of the protein folding process in mammalian host and parasite. A transacetylase activity has been described for calreticulin (reviewed in [32]) and mapped to the P-domain of the protein. Unexpectedly, the described inhibition of glutathione S-transferase (GST) by transacetylation through $H$. contortus calreticulin $[12,13]$ could not be observed in rOvCALR and rMmCALR (Fig. 7). Our data suggests that 7-AMC causes direct acetylation of proteins which in case of GST leads to some activity loss. More important is the observed loss of GST activity caused by calreticulin which is possibly due to chaperoning effects.

Concerning the observed tissue distribution of OvCALR, it can be assumed that the intensely stained cells are highly active in expression of proteins destined for secretion, endosomes, and/or membranes and, therefore, require more calre- 
ticulin than less active or dormant cells. Continuous tegumental membrane turnover [33] and release of proteolytic enzymes from the cecal epithelium [34,35] explain the abundance of calreticulin in these tissues. Confinement of staining to tegumental cell bodies supports the role of OvCALR as endoplasmic chaperone, there was no staining in the cytoplasmic connections to the syncytial tegument or the tegument itself. Likewise, high output of secretory products in accessory glands of the reproductive system and rapid cell divisions at large scale in gametogenesis necessitate high chaperoning activity. Absence of a developed and active reproductive system can also explain the lesser amount of OvCALR RNA in NEJ (Fig. 2) and similar findings were reported for $18 \mathrm{hr}$ schistosomula and cercariae of S. mansoni [9]. It could be expected that suppression of OvCALR will significantly affect development, reproduction, and survival of the parasite in the human host. Indeed, in mouse calreticulin deficiency led to embryonic lethality [36,37]. Remarkably, in Caenorhabditis elegans this was not the case with only reproduction defects observed in mature worms [26]. A later study demonstrated that upregulation of heat shock protein-70 and protein disulfide-isomerase compensated for a loss of calreticulin and/or calnexin in C. elegans [38]. As such it remains to be analyzed whether $O$. viverrini and other trematodes depend on calreticulin/calnexin or have developed a similar safety mechanism. Future analyses could include knock down of calreticulin to determine its importance for the parasite. In addition, we are interested to learn whether OvCALR, if exposed to the host, has any effect on bile duct pathology.

\section{ACKNOWLEDGMENTS}

This work was partially funded by a grant from the Faculty of Allied Health Sciences, Thammasat University in 2015 and through a Royal Golden Jubilee Ph.D. scholarship to Wanlapa Chaibangyang (PHD/0113/2553) jointly funded by the Thailand Research Fund and Thammasat University, Thailand.

\section{CONFLICT OF INTEREST}

All authors declare that they have no conflicts of interest.

\section{REFERENCES}

1. Fliegel L, Burns K, MacLennan DH, Reithmeier RA, Michalak M.
Molecular cloning of the high affinity calcium-binding protein (calreticulin) of skeletal muscle sarcoplasmic reticulum. J Biol Chem 1989; 264: 21522-21528.

2. Smith MJ, Koch GL. Multiple zones in the sequence of calreticulin (CRP55, calregulin, HACBP), a major calcium binding ER/ SR protein. EMBO J 1989; 8: 3581-3586.

3. Munro S, Pelham HR. A C-terminal signal prevents secretion of luminal ER proteins. Cell 1987; 48: 899-907.

4. Michalak M, Groenendyk J, Szabo E, Gold LI, Opas M. Calreticulin, a multi-process calcium-buffering chaperone of the endoplasmic reticulum. Biochem J 2009; 417: 651-666.

5. Raghavan M, Wijeyesakere SJ, Peters LR, Del Cid N. Calreticulin in the immune system: ins and outs. Trends Immunol 2013; 34: 13-21.

6. Lu YC, Weng WC, Lee H. Functional roles of calreticulin in cancer biology. Biomed Res Int 2015; 2015: 526524.

7. de Bruyn M, Wiersma VR, Helfrich W, Eggleton P, Bremer E. The ever-expanding immunomodulatory role of calreticulin in cancer immunity. Front Oncol 2015; 5: 35.

8. Khalife J, Pierce RJ, Godin C, Capron A. Cloning and sequencing of the gene encoding Schistosoma mansoni calreticulin. Mol Biochem Parasitol 1993; 62: 313-315.

9. Khalife J, Liu JL, Pierce R, Porchet E, Godin C, Capron A. Characterization and localization of Schistosoma mansoni calreticulin Sm58. Parasitology 1994; 108: 527-532.

10. Hooker CW, Brindley PJ. Cloning of a cDNA encoding SjIrV1, a Schistosoma japonicum calcium-binding protein similar to calnexin, and expression of the recombinant protein in Escherichia coli. Biochim Biophys Acta 1999; 1429: 331-341.

11. Scott JC, McManus DP. Molecular cloning and functional expression of a cDNA encoding the major endoplasmic reticulumassociated calcium-binding protein, calreticulin, from Philippine strain Schistosoma japonicum. Parasitol Int 1999; 48: 35-46.

12. Singh P, Ponnan P, Krishnan S, Tyagi TK, Priya N, Bansal S, Scumaci D, Gaspari M, Cuda G, Joshi P, Gambhir JK, Saluja D, Prasad AK, Saso L, Rastogi RC, Parmar VS, Raj HG. Protein acyltransferase function of purified calreticulin. Part 1: characterization of propionylation of protein utilizing propoxycoumarin as the propionyl group donor. J Biochem 2010; 147: 625-632.

13. Singh P, Ponnan P, Priya N, Tyagi TK, Gaspari M, Krishnan $S$, Cuda G, Joshi P, Gambhir JK, Sharma SK, Prasad AK, Saso L, Rastogi RC, Parmar VS, Raj HG. Protein acyltransferase function of purified calreticulin: the exclusive role of P-domain in mediating protein acylation utilizing acyloxycoumarins and acetyl CoA as the acyl group donors. Protein Pept Lett 2011; 18: 507517.

14. Young ND, Campbell BE, Hall RS, Jex AR, Cantacessi C, Laha T, Sohn WM, Sripa B, Loukas A, Brindley PJ, Gasser RB. Unlocking the transcriptomes of two carcinogenic parasites, Clonorchis sinensis and Opisthorchis viverrini. PLoS Negl Trop Dis 2010; 4: e719.

15. Rice P, Longden I, Bleasby A. EMBOSS: the European Molecular Biology Open Software Suite. Trends Genet 2000; 16: 276-277. 
16. Sievers F, Wilm A, Dineen D, Gibson TJ, Karplus K, Li W, Lopez R, McWilliam H, Remmert M, Soding J, Thompson JD, Higgins DG. Fast, scalable generation of high-quality protein multiple sequence alignments using Clustal Omega. Mol Syst Biol 2011; 7: 539.

17. Petersen TN, Brunak S, von Heijne G, Nielsen H. SignalP 4.0: discriminating signal peptides from transmembrane regions. Nat Methods 2011; 8: 785-786.

18. Sigrist CJ, de Castro E, Cerutti L, Cuche BA, Hulo N, Bridge A, Bougueleret L, Xenarios I. New and continuing developments at PROSITE. Nucleic Acids Res 2013; 41: 344-347.

19. UniProt Consortium. UniProt: a hub for protein information. Nucleic Acids Res 2015; 43: 204-212.

20. Saito Y, Ihara Y, Leach MR, Cohen-Doyle MF, Williams DB. Calreticulin functions in vitro as a molecular chaperone for both glycosylated and non-glycosylated proteins. EMBO J 1999; 18: 6718-6729.

21. Vichasri-Grams S, Subpipattana P, Sobhon P, Viyanant V, Grams R. An analysis of the calcium-binding protein 1 of Fasciola gigantica with a comparison to its homologs in the phylum Platyhelminthes. Mol Biochem Parasitol 2006; 146: 10-23.

22. Bansal S, Ponnan P, Raj HG, Weintraub ST, Chopra M, Kumari R, Saluja D, Kumar A, Tyagi TK, Singh P, Prasad AK, Saso L, Rastogi RC, Parmar VS. Autoacetylation of purified calreticulin transacetylase utilizing acetoxycoumarin as the acetyl group donor. Appl Biochem Biotechnol 2009; 157: 285-298.

23. Kathuria A, Gupta A, Priya N, Singh P, Raj HG, Prasad AK, Parmar VS, Sharma SK. Specificities of calreticulin transacetylase to acetoxy derivatives of 3-alkyl-4-methylcoumarins: effect on the activation of nitric oxide synthase. Bioorg Med Chem 2009; 17: 1550-1556.

24. Young ND, Nagarajan N, Lin SJ, Korhonen PK, Jex AR, Hall RS, Safavi-Hemami H, Kaewkong W, Bertrand D, Gao S, Seet Q, Wongkham S, Teh BT, Wongkham C, Intapan PM, Maleewong W, Yang X, Hu M, Wang Z, Hofmann A, Sternberg PW, Tan P, Wang J, Gasser RB. The Opisthorchis viverrini genome provides insights into life in the bile duct. Nat Commun 2014; 5: 4378.

25. Baksh S, Michalak M. Expression of calreticulin in Escherichia coli and identification of its $\mathrm{Ca} 2+$ binding domains. J Biol Chem 1991; 266: 21458-21465.

26. Park BJ, Lee DG, Yu JR, Jung SK, Choi K, Lee J, Lee J, Kim YS, Lee JI, Kwon JY, Lee J, Singson A, Song WK, Eom SH, Park CS, Kim DH, Bandyopadhyay J, Ahnn J. Calreticulin, a calcium-binding molecular chaperone, is required for stress response and fertility in Caenorhabditis elegans. Mol Biol Cell 2001; 12: 2835-2845.

27. Leach MR, Cohen-Doyle MF, Thomas DY, Williams DB. Localization of the lectin, ERp57 binding, and polypeptide binding sites of calnexin and calreticulin. J Biol Chem 2002; 277: 2968629697.

28. Raj HG, Kohli E, Goswami R, Goel S, Rastogi RC, Jain SC, Wen- gel J, Olsen CE, Parmar VS. Mechanism of biochemical action of substituted benzopyran-2-ones. Part 8: Acetoxycoumarin: protein transacetylase specificity for aromatic nuclear acetoxy groups in proximity to the oxygen heteroatom. Bioorg Med Chem 2001; 9: 1085-1089.

29. Kozlov G, Pocanschi CL, Rosenauer A, Bastos-Aristizabal S, Gorelik A, Williams DB, Gehring K. Structural basis of carbohydrate recognition by calreticulin. J Biol Chem 2010; 285: 3861238620.

30. Chouquet A, Païdassi H, Ling WL, Frachet P, Houen G, Arlaud GJ, Gaboriaud C. X-ray structure of the human calreticulin globular domain reveals a peptide-binding area and suggests a multimolecular mechanism. PLoS One 2011; 6: e17886.

31. Schrag JD, Bergeron JJ, Li Y, Borisova S, Hahn M, Thomas DY, Cygler M. The Structure of calnexin, an ER chaperone involved in quality control of protein folding. Mol Cell 2001; 8: 633-644.

32. Kumari R, Bansal S, Gupta G, Arora S, Kumar A, Goel S, Singh P, Ponnan P, Priya N, Tyagi TK, Baghel AS, Manral S, Tandon R, Joshi R, Rohil V, Gaspari M, Kohli E, Tyagi YK, Dwarakanath BS, Saluja D, Chatterji S, Sharma SK, Prasad AK, Rastogi RC, Raj HG, Parmar VS. Calreticulin transacylase: genesis, mechanism of action and biological applications. Biochimie 2010; 92: 1173-1179.

33. Apinhasmit W, Sobhon P, Saitongdee P, Menayotin S, Upatham ES. Opisthorchis viverrini: ultrastructure of the tegument of the first-week juveniles and adult flukes. Int J Parasitol 1994; 24: 613-621.

34. Kaewpitoon N, Laha T, Kaewkes S, Yongvanit P, Brindley PJ, Loukas A, Sripa B. Characterization of cysteine proteases from the carcinogenic liver fluke, Opisthorchis viverrini. Parasitol Res 2008; 102: 757-764.

35. Sripa J, Laha T, To J, Brindley PJ, Sripa B, Kaewkes S, Dalton JP, Robinson MW. Secreted cysteine proteases of the carcinogenic liver fluke, Opisthorchis viverrini: regulation of cathepsin F activation by autocatalysis and trans-processing by cathepsin B. Cell Microbiol 2010; 12: 781-795.

36. Mesaeli N, Nakamura K, Zvaritch E, Dickie P, Dziak E, Krause $\mathrm{KH}$, Opas M, MacLennan DH, Michalak M. Calreticulin is essential for cardiac development. J Cell Biol 1999; 144: 857-868.

37. Guo L, Nakamura K, Lynch J, Opas M, Olson EN, Agellon LB, Michalak M. Cardiac-specific expression of calcineurin reverses embryonic lethality in calreticulin-deficient mouse. J Biol Chem 2002; 277: 50776-50779.

38. Lee W, Kim KR, Singaravelu G, Park BJ, Kim DH, Ahnn J, Yoo YJ. Alternative chaperone machinery may compensate for calreticulin/calnexin deficiency in Caenorhabditis elegans. Proteomics 2006; 6: 1329-1339.

39. Frickel EM, Riek R, Jelesarov I, Helenius A, Wuthrich K, Ellgaard L. TROSY-NMR reveals interaction between ERp57 and the tip of the calreticulin P-domain. Proc Natl Acad Sci USA 2002; 99: 1954-1959. 